\title{
Die Rechte zukünftiger Kinder im Kontext pränataler Diagnostik
}

\author{
Dagmar Schmitz $\mathbb{D} \cdot$ Marcus Düwell
}

Eingegangen: 25. Januar 2021 / Angenommen: 9. Juni 2021 / Online publiziert: 2. August 2021

(C) Der/die Autor(en) 2021

Zusammenfassung Das Gendiagnostikgesetz (GenDG) verbietet seit 2010 die pränatale Diagnostik spätmanifestierender Erkrankungen (§ 15(2) GenDG). In seiner Begründung bezog sich der Gesetzgeber in Analogie zu internationalen Empfehlungen für den pädiatrischen Bereich vor allem auf das Recht des heranwachsenden Kindes bzw. des späteren Erwachsenen auf Nichtwissen. Mit diesem gesetzlichen Verbot hat Deutschland einen viel diskutierten Sonderweg in der Regulierung genetischer Pränataldiagnostik eingeschlagen. Seither jedoch hat sich nicht nur die Perspektive auf prädiktive Testungen im Kindesalter verändert. In zunehmendem Maße generieren auf das gesamte Genom abzielende Diagnostikangebote auch andere vorgeburtlich genetische Informationen, die - in ähnlicher Weise wie diejenigen zu spätmanifestierenden Erkrankungen eine potenzielle Gefahr für das Recht auf Nichtwissen der späteren Person darstellen. Es soll daher im Rahmen dieses Beitrages überprüft werden, inwiefern das deutsche Diagnostikverbot und dessen Bezug auf das Recht auf Nichtwissen des späteren Kindes eine tragfähige Basis zur Regulierung gendiagnostischer Untersuchungen in der Pränatalmedizin insgesamt darstellt bzw. zukünftig darstellen kann.

Untersucht wird der Argumentationsgang des Diagnostikverbotes im Hinblick auf spätmanifestierende Erkrankungen vor dem Hintergrund der Entwicklungen in der prädiktiven (genomischen) Diagnostik. Eine Analyse des normativen Begründungsrahmens zeigt, dass nach wie vor deutlicher Klärungsbedarf hinsichtlich der philosophisch-ethischen Fundierung wie auch hinsichtlich der Interpretation eines Rechts auf Nichtwissen eines (zukünftigen) Kindes im Kontext von genetischer Di-

Dr. med. Dagmar Schmitz $(\bowtie)$

Institut für Geschichte, Theorie und Ethik der Medizin, Uniklinik RWTH Aachen,

Wendlingweg 2, 52074 Aachen, Deutschland

E-Mail: daschmitz@ukaachen.de

Dr. phil. Marcus Düwell

Fachbereich Philosophie, Otto-Friedrich Universität Bamberg, Bamberg, Deutschland 
agnostik besteht. Darüber hinaus müssen auch Interessen des (zukünftigen) Kindes an eventuell möglich werdender pränataler Behandlung oder Prävention von Erkrankungen in die Abwägung miteinbezogen werden.

Um zukünftigen Herausforderungen genomischer Pränataldiagnostik und -therapie begegnen zu können, müsste dringend geklärt werden, inwiefern Rechte zukünftiger Personen tatsächlich in der speziellen Situation der Schwangerschaft relevant sein können und mit welchen Pflichten diese für den Gesetzgeber, aber insbesondere auch für zukünftige Eltern und Ärzte einhergehen. Der Menschenrechtsdiskurs könnte hierfür eine tragfähige Basis und hilfreiche Strategien liefern.

Schlüsselwörter Vorgeburtliche Diagnostik - Genetische Untersuchungen · Recht auf eine offene Zukunft · Spätmanifestierende Erkrankung · Recht auf Nichtwissen

\title{
The rights of future children in the context of prenatal testing
}

\begin{abstract}
Definition of the problem Since 2010, the German Act on Genetic Testing (Gendiagnostikgesetz, GenDG) precludes prenatal testing for late-onset diseases. The legislature argued in analogy with professional recommendations for genetic testing of children that the right not to know its own genetic condition has to be protected for the child until she or he is capable of making a decision on her/his own. This law constituted an internationally unique prohibition. More than 10 years later, groundbreaking innovations have sped up the development of new genomic testing procedures and the relevance of the right not to know one's own genetic condition is increasingly questioned in the debate on genomic testing of children. The growing availability of genetic information about the future child will raise the question whether the prohibition has to be extended to other diagnostic procedures. Therefore, a re-examination of this exceptional legal regulation of prenatal genetic testing and its reference to the child's right not to know from an ethical point of view seems to be adequate.

Arguments An analysis of the normative framework of the legal regulation of prenatal testing for late-onset diseases shows that the theoretical foundation of a child's right not to know as well as its interpretation in the context of prenatal genetic testing requires further clarification. In addition, health-related interests or rights of the future child might be increasingly relevant in the context of prenatal testing and therapy and would have to be weighed against a possible right not to know.

Conclusion New advances in prenatal (genomic) testing and therapy ask for a new, comprehensive normative framework which takes into account possible rights of future persons, as well as correlative duties of parents and health care professionals. The human rights discourse could provide a fruitful basis and helpful strategies in this endeavor.
\end{abstract}

Keywords Prenatal testing - Genetic testing - Right to an open future - Late-onset disease $\cdot$ Right not to know 


\section{Einleitung}

In Deutschland ist die Durchführung genetischer Untersuchungen am Menschen seit 2010 durch das Gendiagnostikgesetz (GenDG) geregelt. Während das Gesetz in vielen Bereichen ähnliche Regelungen vorsieht, wie sie auch von anderen Ländern getroffen wurden, hat es jedoch auch ein Alleinstellungsmerkmal: das Verbot einer pränatalen Diagnostik spätmanifestierender (also erst nach dem vollendeten 18. Lebensjahr auftretender) Erkrankungen ( $\$ 15(2)$ GenDG). In seiner Begründung bezieht sich der Gesetzgeber in Analogie zu internationalen Empfehlungen für den pädiatrischen Bereich vor allem auf das Recht des heranwachsenden Kindes bzw. des späteren Erwachsenen auf Nichtwissen im Hinblick auf den eigenen genetischen Status. Dieses Recht könne dann verletzt werden, wenn die Schwangere bzw. das Paar sich bei auffälligem Ergebnis der Pränataldiagnostik dazu entschlösse, die Schwangerschaft fortzuführen. In diesem Fall werde genetisches Wissen generiert, obwohl die Zustimmung des Betroffenen nicht vorliege und so § 14 GenDG (Genetische Untersuchungen bei nicht-einwilligungsfähigen Personen) umgangen (BTDrs 16/12713, S. 35-36). In $§ 14$ wird die Zulässigkeit einer genetischen Untersuchung an nicht-einwilligungsfähigen Personen prinzipiell von ihrem medizinischen Nutzen (im Hinblick auf Vermeidung, Behandlung oder Vorbeugung einer genetisch bedingten Erkrankung) abhängig gemacht. Das Verbot in $\S 15(2)$ impliziert demnach, dass eine pränatale Diagnostik auf spätmanifestierende Erkrankungen keinen medizinischen Nutzen für die Betroffenen hat.

Das erst wenige Tage vor Gesetzesannahme eingefügte Verbot wurde von vielen Selbsthilfeverbänden als wichtiges Instrument zum Schutz der informationellen Selbstbestimmung begrüß $\mathrm{t}^{1}$, stand von Anfang an aber auch in der Kritik. Verwiesen wurde dabei auf medizinische Auslegungsprobleme sowie auf ethische und rechtliche Inkonsistenzen in der Begründung (Krones et al. 2014). Auch traten schnell Fragen im Hinblick auf die Zulässigkeit der seit 2011 in Deutschland möglichen Präimplantationsdiagnostik im Hinblick auf spätmanifestierende Erkrankungen auf. Inwiefern sich der hinter dem Verbot stehende Argumentationsgang auch über den Kontext von spätmanifestierenden Erkrankungen hinaus in den übergeordneten normativen Rahmen für den Umgang mit (genomischer) Pränataldiagnostik integrieren ließe, wurde unseres Wissens bisher nicht untersucht. Spätmanifestierende genetisch bedingte Erkrankungen schienen offensichtlich außergewöhnliche und einzigartige Probleme zu bieten, für die ebensolche Lösungen als erforderlich angesehen wurden.

International wird häufig angenommen, dass es ein Recht auf Nichtwissen des zukünftigen Kindes geben sollte, aber dessen Schutz versucht man im pränataldiagnostischen Kontext auf andere Weise zu realisieren. Der Schwerpunkt liegt dabei eher auf angemessener Beratung der Schwangeren bzw. des Paares. So sehen zum Beispiel die internationalen Empfehlungen zur prädiktiven Testung der Huntington Krankheit eine vorgeburtliche Diagnostik zwar auch als nicht indiziert an, falls das Paar die Schwangerschaft unabhängig vom Testergebnis fortsetzen möchte (MacLeod et al. 2013). Hierüber sollten die Schwangere bzw. das Paar im Rahmen

\footnotetext{
1 https://www.imew.de/de/ethik-forum/spaetmanifeste-krankheiten-gendg/?type=1 (zuletzt aufgerufen am 09. Dez. 2020).
} 
einer genetischen Beratung informiert werden. Ein generelles Verbot von Pränataldiagnostik auf spätmanifestierende Erkrankungen (ebenso wie ein generelles Verbot der prädiktiven Testung Minderjähriger) wird jedoch nicht ausgesprochen (MacLeod et al. 2013; Wadrup et al. 2019). Auch wird die grundsätzliche Zulässigkeit eines derartigen Diagnostikverbotes thematisiert und kontrovers diskutiert (Duncan et al. 2006).

In den letzten 10 Jahren seit Inkrafttreten des Gendiagnostikgesetzes haben sich verschiedene Entwicklungen im Bereich der prädiktiven genetischen Diagnostik vollzogen, die Anlass geben, den grundsätzlichen Argumentationsgang hinter § 15(2) einer erneuten Prüfung zu unterziehen und nun vor allem seine Tauglichkeit im normativen Gesamtkontext der Pränataldiagnostik in den Blick zu nehmen. Insbesondere die ungeheuren Fortschritte, die auf dem Gebiet der (vorgeburtlichen) genomischen Diagnostik erzielt werden konnten, scheinen eine Sonderbehandlung genetischer Informationen im Hinblick auf spätmanifestierende Erkrankungen in Frage zu stellen. Neue Sequenziertechnologien ermöglichen nicht nur das nicht-invasive Screening auf fetale Aneuploidien (Bianchi und Chiu 2018) und die nichtinvasive Diagnostik einzelner monogenetischer fetaler Erkrankungen anhand einer Blutprobe der Schwangeren (Mellis et al. 2018), sondern auch zunehmend die Analyse des gesamten fetalen Exoms bzw. Genoms (Monaghan et al. 2020; Ferretti et al. 2019; Mellis et al. 2018). Eine ungezielte Bereitstellung einer großen Zahl genetischer Informationen über den Fetus wird damit deutlich einfacher und auch kostengünstiger. Die im Kontext von spätmanifestierenden Erkrankungen identifizierten Probleme werden entsprechend auf einer übergeordneten Ebene adressiert werden müssen. Die zeitnahe Verständigung über einen umfassenden normativen Rahmen für pränatale genomische Testungen, der auch die Interessen bzw. Rechte des zukünftigen Kindes (z. B. auf infornmationelle Selbstbestimmung) in den Blick nimmt, wäre damit dringend geboten (Dondorp et al. 2016). Die grundsätzliche Argumentation hinter $\S 15(2)$ ist also daraufhin zu überprüfen, inwiefern sie in einen übergeordneten normativen Rahmen für vorgeburtliche genetische Untersuchungen integrierbar wäre bzw. hierfür sogar eine Basis liefern könnte.

Uns scheint, dass der Argumentationsgang zu $\S 15(2)$ wie folgt rekonstruiert werden kann:

1. Personen haben ein Recht auf informationelle Selbstbestimmung im Hinblick auf ihren genetischen Status ( 1 GenDG).

2. Dieses Recht kann von Kindern noch nicht ausgeübt werden, jedoch bereits in der Kindheit verletzt werden, indem genetische Informationen ohne ihr Einverständnis erzeugt werden ( $\$ 14$ GenDG).

3. Gerechtfertigt werden kann eine derartige Rechtsverletzung durch einen unmittelbaren medizinischen Nutzen (Vermeidung, Behandlung oder Vorbeugung von Erkrankungen) der Diagnostik für die betroffene Person (§ 14 Absatz 1 Satz 1 GenDG).

4. Auch durch vorgeburtliche Diagnostik kann - sollte die Schwangerschaft fortgesetzt werden - das Recht auf informationelle Selbstbestimmung des späteren Erwachsenen verletzt werden ( 15 Absatz 2 GenDG). 
5. Bei einer vorgeburtlichen Diagnostik im Hinblick auf spätmanifestierende Erkrankungen gibt es keinen medizinischen Nutzen für die betroffene Person und daher keine Rechtfertigung für eine derartige Rechtsverletzung.

Wir behaupten, dass diese Rekonstruktion alle Schritte beinhaltet, die zum Verständnis des derzeitigen normativen Rahmens strikt erforderlich sind, d.h. alle fünf Schritte müssen begründet sein, um diesen Rahmen verteidigen zu können. Im Folgenden wollen wir zeigen, dass zumindest drei der zugrundeliegenden Annahmen ((2), (4), (5)) wichtige Fragen aufwerfen, deren Klärung unabdingbar erscheint, bevor eine Integration des Argumentationsganges in einen umfassenderen normativen Rahmen für Pränataldiagnostik gelingen kann. Hierzu werden wir im anschließenden Teil einige Vorschläge unterbreiten.

\section{Kritische Diskussion des Argumentationsganges}

\section{Ad 2) Informationelle Selbstbestimmung und das Recht auf eine offene Zukunft}

Viele Empfehlungen zum Schutz von Rechten von Kindern (nicht allein im medizinischen bzw. gendiagnostischen Kontext) beziehen sich auf den einflussreichen und breit rezipierten Aufsatz des US-amerikanischen Rechtsphilosophen Joel Feinberg (1926-2004) zu dem Recht des Kindes auf eine offene Zukunft (Feinberg 1980). Feinberg unterscheidet hier zwischen moralischen Rechten, die nur Erwachsene haben (A-rights), moralischen Rechten, die Kinder haben $(C$-rights) und moralischen Rechten, die beide Gruppen gemeinsam haben $(A$ - $C$-rights). Unter den $C$-rights betont Feinberg insbesondere das Recht auf eine offene Zukunft als eine Gruppe antizipatorischer Autonomie-Rechte von Kindern ,which are to be saved for the child until he is an adult, but which can be violated ,in advance" so to speak" (Feinberg 1980, S. 125 f.) Zu diesen antizipatorischen Autonomierechten wäre auch das Recht auf informationelle Selbstbestimmung zu zählen. Typische Beispiele für Verletzungen des Kindsrechts auf eine offene Zukunft betreffen Handlungen an Kindern, die aus religiösen Überzeugungen der Eltern erfolgen und weitreichende Folgen für das Kind haben können. Hier kann unter Umständen sogar ein Eingreifen des Staates erforderlich sein. Feinberg erläutert seinen Ansatz exemplarisch anhand von Gerichtsprozessen mit Mitgliedern der Glaubensgemeinschaften „Zeugen Jehovas“ und „Amish“. Andere Autoren diskutieren praktische Implikationen eines solches Rechts für den medizinischen Kontext, u. a. bei genetischen Testungen (Davis 1997) oder nicht-therapeutischen Beschneidungen (Darby 2013).

Von philosophischer Seite wird die Begründung und Anwendung eines solchen Rechts auf offene Zukunft jedoch zunehmend kritisch hinterfragt (Mills 2003; Millum 2014; Chen 2016). Feinberg biete weder ein theoretisches Fundament für ein Kindesrecht auf eine offene Zukunft, noch eine inhaltliche Klärung bzw. Kriterien oder eine Methode zur Identifikation mutmaßlicher Verletzungen eines solchen Rechts. Möglicherweise tragen auch die hier beschriebenen Defizite dazu bei, dass Feinbergs Ansatz seinen Einfluss auf Leitlinien und Empfehlungen in der pädiatrischen Medizin verliert. Während das Kindesrecht auf eine offene Zukunft seit den 
1990er-Jahren viele Richtlinien beispielsweise zu prädiktiver genetischer Diagnostik dominierte (Borry et al. 2006; Garrett et al. 2019), lassen aktuellere Stellungnahmen vermehrt Ausnahmen zu und verlangen eine bessere Gewichtung der verschiedenen Interessen (Botkin et al. 2015; Committee on Bioethics et al. 2013). Die Ungewissheit im Hinblick auf ein späteres Leiden an einer nicht-behandelbaren Erkrankung könne beispielsweise eine schwere psychosoziale Belastung und damit ein großes Interesse an einer genetischen Untersuchung mit sich bringen (Botkin et al. 2015). Außerdem impliziere Elternschaft im Allgemeinen unvermeidlich Handlungen, die das Kind in seinen zukünftigen Entscheidungen einschränkten. Die Forderung nach einem Recht auf eine offene Zukunft sei daher praxisfern, mehrdeutig und missverständlich (Garrett et al. 2019). Es wird angeregt, den Rechtsbegriff und damit die Absolutheit seines Geltungsanspruches zu verlassen, und stattdessen das Interesse eines Kindes an einer (möglichst) offenen Zukunft als eines unter mehreren relevanten Interessen anzusehen (Garrett et al. 2019), so dass eine Abwägung im Einzelfall möglich wird.

Auch wenn dies Konzept der offenen Zukunft in der Ausarbeitung von Feinberg Fragen aufwirft, kann man die diesem zugrundeliegenden moralischen Überlegung nicht vermeiden, wenn man bestimmte Aspekte unseres Umgangs mit Kindern verständlich machen will. So setzen etwa das Recht auf Bildung oder die Praxis medizinischer Frühversorgung voraus, dass es verwerflich ist, ein Kind bezüglich seiner Möglichkeiten in der Zukunft zu schädigen. Ein Recht auf Bildung kann man nur dann annehmen, wenn man annimmt, dass das Kind ein Recht darauf hat, in der Zukunft bestimmte Handlungs- und Entwicklungsmöglichkeiten zu haben. Das bedeutet: Wie immer man dieses Recht auf eine offene Zukunft auch im Detail ausarbeitet, man muss zumindest im Grundsatz ein solches Recht annehmen, wenn es plausibel sein soll, dass Handlungen an Neugeborenen und Kindern verboten bzw. geboten sind, auch wenn diese Handlungen das Kind nicht in seinen aktuellen Rechten beeinträchtigen. Rechte von Neugeborenen und Kindern müssen stets eine auf Zukunft angelegte Dimension haben, die deren aktuelle Interessen überschreitet.

Das fordert nun im Hinblick auf die Regelung der Humangenetik eine Diskussion, die im deutschen Regelungskontext bisher - soweit uns bekannt - nicht geführt wurde. Auch in den Stellungnahmen der deutschen Gesellschaft für Humangenetik zur genetischen Diagnostik bei Kindern findet sich lediglich der Hinweis auf das GenDG und die entsprechenden Richtlinien der Gendiagnostikkommission. ${ }^{2}$ Es wäre also geboten, die international begonnene Debatte auch im deutschen Kontext weiterzuführen und ggfs. den Feinbergschen Ansatz in den kritischen Begründungsaspekten weiterzuentwickeln, bevor dessen Grundidee in den normativen Rahmen (genomischer) Präntaldiagnostik übernommen werden kann.

\section{Ad 4) Vorgeburtliche Rechtsverletzungen?}

Eine Übertragung des Feinbergschen Ansatzes in den vorgeburtlichen Bereich, wie es das GenDG zu tun scheint, ist noch komplexer, insofern dieser Schritt auf eine Rei-

\footnotetext{
${ }^{2}$ https://www.gfhev.de//de/veroeffentlichungen/leitlinien_stellungnahmen.html (zuletzt aufgerufen am 09. Dez. 2020).
} 
he von keineswegs unstrittigen Voraussetzungen aufbaut. In der wissenschaftlichen Diskussion wurde dieser Transfer bisher nur vereinzelt vorgenommen. Davis diskutierte beispielsweise den Fall eines gehörlosen Paares, das um Pränataldiagnostik bat, um sicherzustellen, dass das Kind ebenfalls gehörlos sein würde (Davis 1997). Eine derartige Wahl würde die Zukunft eines Kindes nach Ansicht der Autorin auf unangemessene Weise einschränken. Völlig unklar ist jedoch zunächst einmal, ob der Ansatz Feinbergs überhaupt auf die vorgeburtliche Situation übertragbar ist. In der (frühen) Kindheit existiert der Rechtsträger bereits, ist jedoch noch nicht in der Lage, sein Recht auch auszuüben. Im vorgeburtlichen Bereich kann die Existenz des Rechtsträgers demgegenüber noch nicht als selbstverständlich gegeben angenommen werden. Wir sind uns natürlich bewusst, dass diese Frage in vielfacher Hinsicht umstritten ist. Für unseren Diskussionskontext können wir jedoch festhalten, dass damit wichtige Voraussetzungen von moralischen Verpflichtungen gegenüber dem zukünftigen Rechtsträger derart tangiert sind, dass man nicht einfach davon ausgehen kann, dass die gleichen Rechte zugeschrieben werden können, wie dies bei Kindern der Fall ist. Bevor ein derartiges Verbot ausgesprochen werden kann, müsste also zunächst geklärt werden, ob es tatsächlich moralische Rechte zukünftiger Kinder und Personen gibt, die in der Pränatalmedizin relevant sein könnten.

Eine Debatte zu moralischen Rechten und korrespondierenden Verpflichtungen setzt normalerweise die Existenz des Rechtsträgers voraus. Aber die aktuelle Debatte zur Generationengerechtigkeit zeigt, dass es nicht unmöglich ist, moralische Verpflichtungen gegenüber zukünftigen Personen anzunehmen, auch wenn diese noch nicht existieren. Obwohl wir nicht wissen, ob und in welcher Zahl es sie einmal geben wird, wird doch die Frage nach moralischen Verpflichtungen gegenüber zukünftigen Generationen u. a. im Kontext des Klimawandels zunehmend wichtiger. Möchte man diese Verpflichtungen in einem Rechte-basierten Rahmen diskutieren, dann ergeben sich mehrere Schwierigkeiten. Insbesondere das Nicht-Existenzproblem und das Nicht-Identitätsproblem werden hierzu diskutiert (Gosseries 2008; Unruh 2016). Ersteres thematisiert die Schwierigkeit, nicht-existenten Personen Rechte zuzusprechen. Viele Autoren stimmen darin überein, dass zukünftige Personen keine Rechtsträger in der Gegenwart sind, vertreten aber unterschiedliche Positionen hinsichtlich der Frage, ob zukünftige Rechte zukünftiger Menschen mit korrelierenden Verpflichtungen für aktuell lebende Menschen einhergehen können. Diejenigen, die von solchen Verpflichtungen ausgehen, argumentieren beispielsweise, dass ein Bekenntnis zu den Menschenrechten impliziert, dass die grundlegenden Voraussetzungen der Bedingung der Handlungsfähigkeit für alle Menschen geschützt werden. Ein solches Bekenntnis ist jedoch nur dann plausibel, wenn auch diese Bedingungen für zukünftige Menschen geschützt werden, sofern wir Gründe haben anzunehmen, dass es solche Menschen geben wird. Wenn das (wie im pränataldiagnostischen Kontext mit großer Wahrscheinlichkeit) der Fall ist, geht ein Bekenntnis zu den Menschenrechten notwendigerweise einher mit Verpflichtungen gegenüber den zukünftigen Rechten zukünftiger Menschen (Düwell und Bos 2016).

Das zweite Problem bezieht sich auf die Frage, ob Personen Schaden zugefügt werden kann durch Handlungen, deren Ausführung diese Personen ihre Existenz überhaupt erst verdanken. Ist es zum Beispiel möglich, durch Pränataldiagnostik das Recht eines zukünftigen Kindes auf Nichtwissen im Hinblick auf seinen geneti- 
schen Status zu verletzen, wenn das Paar bzw. die Schwangere die Schwangerschaft in jedem Fall beendet hätten, falls ihnen - wie aktuell in Deutschland der Fall die Möglichkeit einer vorgeburtlichen Diagnostik auf eine spätmanifestierende Erkrankung verwehrt wird? Würde das zukünftige Kind es nicht bevorzugen, dass sein Recht auf Nichtwissen verletzt wird, wenn die einzige Alternative dazu wäre, überhaupt nicht geboren zu werden? Und wenn das Kind durch die Pränataldiagnostik nicht Schaden nehmen kann (weil es ohne Diagnostik nicht zu seiner Existenz gekommen wäre), wer dann? Derek Parfit hat 1984 das Nicht-Identitätsproblem besonders prominent formuliert (Parfit 1984) und damit den Ausgangspunkt zahlloser philosophischer Veröffentlichungen (Roberts und Wasserman 2009; McMahan 2013; Boonin 2014) geliefert. Eine abschließende Bewertung der Konsequenzen für den normativen Rahmen von Pränataldiagnostik, insbesondere auch in seinem Bezug zu den Rechten des zukünftigen Kindes, steht noch aus (Steigleder 1998).

Natürlich kann die Diskussion zu Rechten zukünftiger Generationen auch nicht ohne Weiteres auf die vorgeburtliche Situation übertragen werden: Der Fetus steht in individueller Verbindung zu dem Kind, zu dem er sich entwickeln wird, während es für die Diskussion zur Generationengerechtigkeit nicht wichtig ist, aus welchen Personen die zukünftigen Generationen bestehen werden. Im Hinblick auf die Generationengerechtigkeit ist es sogar wesentlich, dass hier Rechtsgüter zu schützen sind (saubere Luft, sichere Atmosphäre etc.), die generell für Menschen wichtig sind, die zu schützen also verpflichtend ist, unabhängig von spezifischen Charakteristika zukünftiger Generationen, so lange diese grundlegende biologische Eigenschaften mit uns teilen (wie etwa die Angewiesenheit auf Luft zum Atmen etc.). Argumentationsstrukturen aus dem Kontext der Generationengerechtigkeit können sich jedoch unter Umständen als hilfreich erweisen, wenn Rechte zukünftiger Kinder in der Pränatalmedizin (wie beispielsweise das Recht auf informationelle Selbstbestimmung) zur Diskussion stehen. Das bedeutet, dass die in der Diskussion um Generationengerechtigkeit identifizierten Probleme auch für den Pränatalbereich besprochen werden müssen. Nur dann kann der daraus resultierende normative Rahmen die Basis für belastbare und nachhaltige Forderungen liefern.

\section{Ad 5) Der Fetus als Patient}

Das GenDG argumentiert in Bezug auf das Recht auf informationelle Selbstbestimmung grundsätzlich, wie aufgezeigt, in einer Analogie zwischen der Situation in der Kindheit ( $\$ 14$ Genetische Untersuchungen bei nicht-einwilligungsfähigen Personen) und der vorgeburtlichen Situation (§ 15 Vorgeburtliche genetische Untersuchungen). Während jedoch in $\S 14$ eine Abwägung zwischen dem Recht auf informationelle Selbstbestimmung einerseits und relevanten Gesundheitsinteressen des betroffenen Kindes andererseits vorgenommen wird (§ 14 Absatz 1 Satz 1 GenDG), ist dies in der vorgeburtlichen Situation nicht vorgesehen. Stattdessen wird ein absolutes Verbot einer Untergruppe an diagnostischen Maßnahmen ausgesprochen, von der - so lässt sich mutmaßen - kein irgendwie gearteter gesundheitlicher Nutzen im Sinne einer Behandlung, Vorbeugung oder Vermeidung einer Erkrankung erwartet wurde. Eine Abwägung in dieser Hinsicht schien dem Gesetzgeber offensichtlich nicht erforderlich. 
Die Entwicklung der letzten zehn Jahre lassen jedoch hieran Zweifel aufkommen. Zwar diente die Pränataldiagnostik in Ermangelung therapeutischer oder präventiver Optionen traditionell vor allem der reproduktiven Autonomie der Schwangeren. Nun aber scheint sich dieser Fokus zu verschieben (Bianchi 2012; Dukhovny und Norton 2018). Dank bahnbrechender Fortschritte im Bereich der fetalchirurgischen und auch der nicht-chirurgischen Prä- und Perinatalmedizin erleben wir die Begründung einer fetalen Medizin, ausgerichtet auf das Wohl des Feten bzw. des zukünftigen Kinde (Chitty et al. 2020). So ist beispielsweise die pränatale Korrektur einer Spina bifida (Spaltbildung im Bereich der Wirbelsäule, „offener Rücken“) mittlerweile ein etabliertes Verfahren in ausgewählten Fällen (Moldenhauer und Flake 2019). Viele Studien versuchen aktuell, den Nutzen einer pränatalen Intervention für weitere Fehlbildungen etwa im kardialen Bereich und bei Zwerchfellhernien zu belegen (Wenstrom und Carr 2014; Levy et al. 2018). Erste Erfolge gibt es im Bereich der pränatalen molekularen Stammzell- und Gentherapie. Eine deutsche Arbeitsgruppe hat die erfolgreiche pränatale Behandlung von drei Feten mit einer X-chromosomalen Form der ektodermalen Dysplasie publiziert (Schneider et al. 2018). Die vorgeburtliche Gentherapie hat im Tierversuch bei Morbus Gaucher ermutigende Ergebnisse gezeigt (Massaro et al. 2018) und wird darüber hinaus für vielversprechend etwa bei hämatologischen Erkrankungen oder bei zystischer Fibrose gehalten (Almeida-Porada et al. 2019; Carlon et al. 2017). Und auch die pränatale Genomeditierung scheint in greifbare Nähe gerückt zu sein (Rossidis et al. 2018). Obwohl konkrete klinische Forschung zu pränatalen Therapien bei genetisch bedingten Erkrankungen derzeit noch die Ausnahme ist, so scheint doch zumindest eine zukünftige Verfügbarkeit präventiver oder therapeutischer pränataler Interventionen auch für die heterogene Gruppe genetisch bedingter Erkrankungen nicht ausgeschlossen und sicherlich doch als großer Fortschritt zu begrüßen. In einer solchen Situation würde ein Diagnostikverbot eine deutliche Benachteiligung der Betroffenen zur Folge haben und könnte sich dadurch als unhaltbar erweisen.

Es wäre also im pränataldiagnostischen Kontext grundsätzlich erforderlich, eine Abwägung vorzunehmen zwischen dem Recht auf informationelle Selbstbestimmung einerseits und relevanten Gesundheitsinteressen andererseits, die ebenfalls Gegenstand von Rechtsansprüchen sein können. Dass eine solche Abwägung in der Regelung der vorgeburtlichen genetischen Diagnostik für spätmanifestierende Erkrankungen nicht zu finden ist, erscheint als Inkonsistenz in der Argumentation des GenDG. Zumindest wäre zu begründen, warum eine solche Abwägung nicht erforderlich oder nicht statthaft ist. In einem übergeordneten, genomischen Diagnostikkontext erscheint der Verzicht auf Abwägung kaum haltbar.

\section{Mögliche Eckpunkte eines angemessenen normativen Konzeptes}

Wir haben bislang vornehmlich gezeigt, welche Desiderate die bisherige Diskussion aufweist. Im nächsten Schritt soll skizziert werden, in welche Richtung sich die normative Diskussion zukünftig bewegen könnte. Zunächst muss kurz begründet werden, warum diese normativen Fragen als „Rechts“-Konflikte konzipiert werden. Man könnte ja die Auffassung vertreten, dass alle Fragen, die sich Feinberg 
aufbürdet, vermeidbar wären, wenn man die ethischen Fragen nicht auf der Basis von Rechten thematisiert. Auch wird bisweilen vermutet, dass mit der Wahl eines Konzepts von „Rechten“ bereits eine problematische oder zumindest strittige (etwa atomistische) Sozialphilosophie verbunden ist: Ist nicht die ganze Vorstellung irreführend, dass das zukünftige Kind einen Rechtsanspruch hat, der im Zweifelsfall gegen die Schwangere durchgesetzt werden kann? Wir gehen hier nicht davon aus, dass die Konzeption von Rechten notwendigerweise diese Implikationen hat. Es wird auch nicht angenommen, dass Rechte stets ,,absolute“ Rechte sind, die also Abwägungsverbote enthalten. Abwägungen sind schon deshalb erforderlich, weil es ja „Rechte“ im Plural gibt und daher notwendigerweise Gewichtungen möglich sein müssen. Die Konzeptualisierung im Sinne von Rechten ist aber erforderlich, wenn die Diskussion sich im Rahmen einer menschenrechtlichen Ordnung abspielen soll. Die menschenrechtliche Ordnung ist zum einen in der globalen Ordnung, die nach dem Zweiten Weltkrieg entstanden ist, faktisch der rechtlich-politische Bezugsrahmen für normative Auseinandersetzungen; auch zentrale medizinethische Konzepte wie Patientenautonomie, informed consent etc. gehören in diesen Rahmen. Zum anderen gibt es aber auch gute Gründe, in der Entwicklung dieser normativen Ordnung eine moralische Errungenschaft zu sehen. Eine menschenrechtliche Ordnung beinhaltet Freiheitsrechte, deren Einschränkung nur auf der Basis anderer Rechtsgüter möglich ist. Die Diskussion über diese Rechtsgüter ist nicht lediglich eine positivrechtliche Frage, sondern ist insofern eine ethische Frage, als es hier um den normativen Rahmen der rechtlichen Diskussion geht.

Man kann dies sowohl als Skizze einer möglichen Argumentation lesen als auch als eine Agenda für eine zukünftige Diskussion. Dabei deuten wir positive Argumentationslinien an, ohne diese hier ausgiebig besprechen oder begründen zu können.

\section{Rechte des zukünftigen Kindes können antizipatorisch betroffen sein}

Die Diskussion zur Generationengerechtigkeit lehrt uns unseres Erachtens, dass es prinzipiell möglich ist, davon auszugehen, dass Rechtsgüter betroffen sein können, auch wenn der Rechtsträger aktuell noch nicht existiert, wenn er noch nicht als eigenständiger Rechtsträger existiert oder wenn sein Status als Rechtsträger umstritten ist. Dabei muss man sich allerdings bewusst sein, dass es für diese Rechte erforderlich ist zu begründen, warum sie auch unabhängig von spezifischen, jetzt noch unbekannten Wünschen und Interessen zukünftiger Rechtsträger angenommen werden müssen.

Es zeichnet die antizipatorischen Rechte aus, dass sie auch dann Verpflichtungen begründen können, wenn man hinsichtlich des Status des Embryos/Fetus nicht davon ausgeht, dass ein eigener Lebensrechtsanspruch vorliegt. Man kann also Verpflichtungen im Umgang mit Embryonen und Feten formulieren, ohne in der strittigen Frage ihres Status Stellung beziehen zu müssen. Das grenzt aber die Reichweite dieser Argumente insoweit ein, als daraus selbst noch kein Anspruch auf das Lebensrecht erfolgt. Der Anspruch auf diese Rechtsgüter ist insofern hypothetisch, als er davon abhängt, dass die den Fetus betreffenden Handlungen Effekte haben auf eine spätere Person. Dies wäre nicht der Fall, wenn ein Schwangerschaftsabbruch vorgenommen würde. Daraus kann unseres Erachtens jedoch nicht abgeleitet werden, dass 
beispielsweise eine pränatale Diagnostik auf spätmanifestierende Erkrankungen immer dann unproblematisch ist, wenn die Schwangere plant, die Schwangerschaft im Falle eines auffälligen Befundes abzubrechen (wie es die Praxis in anderen Ländern zu sein scheint (Wadrup et al. 2019)). Eine derartige, im Vorfeld der Diagnostik gegebene Zusage kann in ihrer Umsetzung nicht sichergestellt (Wadrup et al. 2019) oder gar erzwungen werden. Es bleibt also unklar, inwiefern die Diagnostik tatsächlich Effekte auf die Rechte der zukünftigen Person haben wird. Zudem wird die Einforderung eines derartigen Versprechens der akut belasteten Lebenssituation der Schwangeren wenig gerecht. Eine Ausarbeitung des normativen Rahmens für vorgeburtliche genetische Diagnostik wird diesen spezifischen Charakter antizipatorischer Rechte aber in jedem Fall berücksichtigen müssen.

\section{Gesundheitsinteressen des zukünftigen Kindes können einen Rechtsanspruch begründen}

Die Zunahme der vorgeburtlichen diagnostischen und insbesondere der therapeutischen Möglichkeiten macht es erforderlich, Gesundheitsinteressen zu identifizieren, die unter Umständen analog zur Situation in der (frühen) Kindheit gegen ein Recht auf Nicht-Wissen abzuwägen wären (wie etwa beim Neugeborenenscreening). Prinzipiell könnte hierbei demnach auf entsprechende Überlegungen aus dem pädiatrischen Bereich zurückgegriffen werden (Burke und Clarke 2016; Gyngell et al. 2019). Es scheint evident angesichts zunehmender genomdiagnostischer Möglichkeiten, dass eine isolierte Betrachtung der Testung auf spätmanifestierende Erkrankungen im Hinblick auf den Schutz der Rechte des zukünftigen Kindes wenig sinnvoll wäre. Das Recht auf informationelle Selbstbestimmung eines zukünftigen Kindes würde seines Kerns beraubt, wenn etwa das gesamte fetale Genom analysiert werden würde (Dondorp et al. 2016). Das Besondere der vorgeburtlichen Situation ist jedoch, dass diagnostische Maßnahmen prinzipiell zwei Ziele verfolgen können: Zusätzlich zu den möglichen Gesundheitsinteressen des zukünftigen Kindes kann hier die reproduktive Autonomie der Schwangeren relevant sein (double-purpose screening (Dondorp und De Wert 2018)), (Narayanan et al. 2018; Richardson und Ormond 2018). Eine klare Unterscheidung der jeweiligen klinischen Pfade und der entsprechenden normativen Rahmenbedingungen in Abhängigkeit von dem mit der Diagnostik verfolgten Ziel erscheint daher dringend erforderlich. Dabei würde eine solche Ausarbeitung zudem den Effekt haben, dass überhaupt deutlich wird, was genau der Gegenstand der ,reproduktiven Autonomie“ sein kann bzw. sein sollte. Es scheint ersichtlich, dass die Forderung nach reproduktiver Autonomie nicht die Inanspruchnahme aller denkbaren Diagnosemöglichkeiten impliziert. Eine nähere Bestimmung der Grenzen des Akzeptablen aber kann nur im Licht konkreter klinischer Möglichkeiten gelingen.

\section{Aus den Rechten zukünftiger Kinder erwachsen Verpflichtungen für die Medizin}

Falls es zutrifft, dass der derzeitige normative Rahmen der rechtlichen Regelung der vorgeburtlichen genetischen Diagnostik (nicht nur, aber auch) im Hinblick auf 
spätmanifestierende Erkrankungen nicht länger adäquat ist, dann ist es auch eine Aufgabe der ärztlichen Profession, sich an der Neugestaltung zu beteiligen. Da mit Blick auf die Entwicklungen der letzten zehn Jahre der bisherige Rückzug auf die reproduktive Autonomie der Schwangeren als alleinige oder überwiegende Legitimation pränataldiagnostischer Maßnahmen an seine Grenzen kommt (bzw. einer näheren Bestimmung bedarf) (Schmitz 2013), wird eine Neu-Positionierung aus professionsethischer Sicht unumgänglich. Das Bewusstsein hierfür scheint auch in der professionsethischen Debatte zunehmend zu wachsen, wie der aktuelle Diskurs zur Kassenfinanzierung der nicht-invasiven Pränataltestung auf Trisomie 21 illustriert (Schmitz 2019; Iacobucci 2018). Ob es allerdings ganz in der Hand der ärztlichen Profession liegen sollte, den Zugang zu fetalen genomischen Untersuchungen zu regulieren (Berkman und Bayefsky 2017), bleibt zu bezweifeln. Angesichts der involvierten Rechtsgüter handelt es sich aus unserer Sicht eher um eine gesamtgesellschaftliche Aufgabe.

\section{Handlungsoptionen könnten ein Abwägungskriterium sein}

Im Unterschied zu den antizipatorischen Rechten des zukünftigen Kindes handelt es sich im Falle der Schwangeren um die Rechte einer Person, die konkrete Wünsche und Interessen hat und diese in der Regel auch artikulieren kann. Abwägungskriterien müssen daher dieser besonderen, ungleichen Ausgangssituation gerecht werden. Wie oben bereits angesprochen, bietet der Menschenrechtsdiskurs einen Rahmen, der die Diskussion antizipatorischer Rechte ermöglicht. Ein möglicher Kandidat für ein Hierarchisierungsprinzip wäre das Principle of Generic Consistency $(P G C)$, wonach sich die Hierarchie von Rechtsgütern daraus ergibt, inwieweit grundlegende Bedingungen der Handlungsfähigkeit des Betroffenen berührt sind (Gewirth 1978; Beyleveld et al. 2015). Abwägungen betreffen dabei nicht nur die grundsätzliche Zulässigkeit einer diagnostischen Maßnahme, sondern in einem weiteren Schritt auch deren Finanzierung im Rahmen eines Gesundheitssystems. Auch in diesem Bereich wurden bereits Überlegungen zu einer Neuordnung angestellt, die den genomdiagnostischen und fetaltherapeutischen Entwicklungen der letzten zehn Jahre Rechnung trägt und unter diesen neuen Bedingungen eine gerechte Ressourcenverteilung ermöglichen soll (Stapleton et al. 2019).

\section{Ausblick}

Wir haben unsere Analyse begonnen mit der Frage, ob das im GenDG verankerte Verbot der pränatalen Diagnostik für spätmanifestierende Erkrankungen vor dem Hintergrund der Entwicklungen der letzten zehn Jahre ein tragfähiges Fundament für den Schutz der informationellen Selbstbestimmung im übergeordneten Kontext der (genomischen) Pränataldiagnostik liefern könnte. Aufgrund der fehlenden theoretischen Ausarbeitung, der damit verbundenen Unklarheiten zur Übertragbarkeit auf den pränatalen Kontext und zu möglichen Abwägungskriterien (z. B. auch im Hinblick auf Gesundheitsinteressen des zukünftigen Kindes) scheint der derzeitige normative Rahmen des Diagnostikverbotes keine angemessene Basis für die anste- 
henden Herausforderungen der Pränatalmedizin zu bieten. In Anknüpfung an den Menschenrechtsdiskurs könnte aus unserer Sicht jedoch eine überzeugende Fundierung und Integration in ein normatives Gesamtkonzept für Pränataldiagnostik gelingen.

Überaus vorausschauend mutet es aus heutiger Perspektive allerdings an, dass mit diesem Verbot erstmals die Rechte des zukünftigen Kindes bzw. der zukünftigen Person in einer Weise Eingang in den Regelungskontext von Pränataldiagnostik gefunden haben, die über reine Lebensrechts- und Lebensschutzerwägungen hinausgeht.

Die Diskussion, die wir hier anstoßen, berührt nicht allein normative Aspekte juristischer und medizinischer Regelungsfragen. Diese Diagnosemöglichkeiten können sich noch viel umfassender auf unser Selbstverständnis auswirken, insofern als Schwangere sich fragen müssen, wie sie mit ihrer Schwangerschaft umgehen sollen, die Medizin sich rechtfertigen muss für ihre Entscheidungen, ein Gesundheitssystem über Prioritäten in der Finanzierung nachdenken muss und eine Gesellschaft vor weitgehenden Entscheidungen steht über Begrenzungen diagnostischer Angebote. Es wäre angemessen, sich die Reichweite dieses Themas bewusst zu machen und die Frage in der gebotenen Grundsätzlichkeit zu diskutieren.

Funding Open Access funding enabled and organized by Projekt DEAL.

Open Access Dieser Artikel wird unter der Creative Commons Namensnennung 4.0 International Lizenz veröffentlicht, welche die Nutzung, Vervielfältigung, Bearbeitung, Verbreitung und Wiedergabe in jeglichem Medium und Format erlaubt, sofern Sie den/die ursprünglichen Autor(en) und die Quelle ordnungsgemäß nennen, einen Link zur Creative Commons Lizenz beifügen und angeben, ob Änderungen vorgenommen wurden.

Die in diesem Artikel enthaltenen Bilder und sonstiges Drittmaterial unterliegen ebenfalls der genannten Creative Commons Lizenz, sofern sich aus der Abbildungslegende nichts anderes ergibt. Sofern das betreffende Material nicht unter der genannten Creative Commons Lizenz steht und die betreffende Handlung nicht nach gesetzlichen Vorschriften erlaubt ist, ist für die oben aufgeführten Weiterverwendungen des Materials die Einwilligung des jeweiligen Rechteinhabers einzuholen.

Weitere Details zur Lizenz entnehmen Sie bitte der Lizenzinformation auf http://creativecommons.org/ licenses/by/4.0/deed.de.

\section{Einhaltung ethischer Richtlinien}

Interessenkonflikt D. Schmitz und M. Düwell geben an, dass kein Interessenkonflikt besteht.

Ethische Standards Für diesen Beitrag wurden von den Autor*innen keine Studien an Menschen oder Tieren durchgeführt. Für die aufgeführten Studien gelten die jeweils dort angegebenen ethischen Richtlinien.

\section{Literatur}

Almeida-Porada G, Waddington SN, Chan JKY, Peranteau WH, MacKenzie T, Porada CD (2019) In utero gene therapy consensus statement from the IFeTIS. Mol Ther 27(4):705-707. https://doi.org/10.1016/ j.ymthe.2019.02.015

Berkman BE, Bayefsky M (2017) Prenatal whole genome sequencing: an argument for professional selfregulation. Am J Bioeth 17(1):26-28. https://doi.org/10.1080/15265161.2016.1251653 
Beyleveld D, Düwell M, Spahn A (2015) Why and how should we represent future generations in policy making? Jurisprudence 6(3):549-566

Bianchi DW (2012) From prenatal genomic diagnosis to fetal personalized medicine: progress and challenges. Nat Med 18(7):1041-1051. https://doi.org/10.1038/nm.2829

Bianchi DW, Chiu RWK (2018) Sequencing of circulating cell-free DNA during pregnancy. N Engl J Med 379(5):464-473. https://doi.org/10.1056/NEJMra1705345

Boonin D (2014) The non-identity problem and the ethics of future people. Oxford University Press, New York

Borry P, Stultiens L, Nys H, Cassiman JJ, Dierickx K (2006) Presymptomatic and predictive genetic testing in minors: a systematic review of guidelines and position papers. Clin Genet 70(5):374-381. https:// doi.org/10.1111/j.1399-0004.2006.00692.x

Botkin JR, Belmont JW, Berg JS, Berkman BE, Bombard Y, Holm IA, Levy HP, Ormond KE, Saal HM, Spinner NB, Wilfond BS, McInerney JD (2015) Points to consider: ethical, legal, and psychosocial implications of genetic testing in children and adolescents. Am J Hum Genet 97(1):6-21. https://doi. org/10.1016/j.ajhg.2015.05.022

Burke K, Clarke A (2016) The challenge of consent in clinical genome-wide testing. Arch Dis Child 101(11):1048-1052. https://doi.org/10.1136/archdischild-2013-304109

Carlon MS, Vidovic D, Birket S (2017) Roadmap for an early gene therapy for cystic fibrosis airway disease. Prenat Diagn 37(12):1181-1190. https://doi.org/10.1002/pd.5164

Chen J (2016) The right to self-development: an addition to the child's right to an open future. J Soc Philos 47(4):439-456

Chitty LS, Ghidini A, Deprest J, Van Mieghem T, Levy B, Hui L, Bianchi DW (2020) Right or wrong? Looking through the retrospectoscope to analyse predictions made a decade ago in prenatal diagnosis and fetal surgery. Prenat Diagn 40(13):1627-1635. https://doi.org/10.1002/pd.5870

Committee On Bioethics, Committee On Genetics, American College Of Medical Genetics, Genomics S, Ethical, Legal Issues C (2013) Ethical and policy issues in genetic testing and screening of children. Pediatrics 131(3):620-622. https://doi.org/10.1542/peds.2012-3680

Darby RJ (2013) The child's right to an open future: is the principle applicable to non-therapeutic circumcision? J Med Ethics 39(7):463-468. https://doi.org/10.1136/medethics-2012-101182

Davis DS (1997) Genetic dilemmas and the child's right to an open future. Hastings Cent Rep 27(2):7-15

Dondorp W, De Wert G (2018) The 'normalization' of prenatal screening: prevention as prenatal beneficence. In: Schmitz D, Clarke A, Dondorp W (Hrsg) The fetus as a patient: a contested concept and its normative implications. Routledge, London, New York, S 144-153

Dondorp WJ, Page-Christiaens GC, de Wert GM (2016) Genomic futures of prenatal screening: ethical reflection. Clin Genet 89(5):531-538. https://doi.org/10.1111/cge.12640

Dukhovny S, Norton ME (2018) What are the goals of prenatal genetic testing? Semin Perinatol 42(5):270-274. https://doi.org/10.1053/j.semperi.2018.07.002

Duncan RE, Foddy B, Delatycki MB (2006) Refusing to provide a prenatal test: can it ever be ethical? BMJ 333(7577):1066-1068. https://doi.org/10.1136/bmj.38950.645799.55

Düwell M, Bos G (2016) Human rights and future people-possibilities of argumentation. J Hum Rights 15(2):231-250

Feinberg J (1980) The child's right to an open future. In: Aiken W, LaFolette H (Hrsg) Whose child? Children's rights, parental authority and state power. Littlefield, Adams \& Co, Totowa, S $125 \mathrm{f}$

Ferretti L, Mellis R, Chitty LS (2019) Update on the use of exome sequencing in the diagnosis of fetal abnormalities. Eur J Med Genet 62(8):103663. https://doi.org/10.1016/j.ejmg.2019.05.002

Garrett JR, Lantos JD, Biesecker LG, Childerhose JE, Chung WK, Holm IA, Koenig BA, McEwen JE, Wilfond BS, Brothers K, Clinical Sequencing Exploratory Research Consortium Pediatrics Working G (2019) Rethinking the "open future" argument against predictive genetic testing of children. Genet Med 21(10):2190-2198. https://doi.org/10.1038/s41436-019-0483-4

Gewirth A (1978) Reason and morality. University of Chicago Press, Chicago

Gosseries A (2008) On future generations' future rights. J Political Philosophy 16(4):446-474

Gyngell C, Newson AJ, Wilkinson D, Stark Z, Savulescu J (2019) Rapid challenges: ethics and genomic neonatal intensive care. Pediatrics 143(Suppl 1):S14-S21. https://doi.org/10.1542/peds.2018-1099D

Iacobucci G (2018) Non-invasive prenatal testing: public and doctors should be consulted, says BMA. BMJ 362:k2916. https://doi.org/10.1136/bmj.k2916

Krones T, Körner U, Schmitz D, Henn W, Wewetzer C, Kreß H, Netzer C, Thorn P, Bockenheimer-Lucius G (2014) Das Verbot der pränatalen Diagnostik spätmanifestierender Erkrankungen im deutschen Gendiagnostikgesetz - eine Diskussion medizinischer und rechtlicher Aspekte und deren Implikati- 
on für die medizinethische Diskussion. Ethik Med 26(1):33-46. https://doi.org/10.1007/s00481-0120240-6

Levy B, Bianchi DW, Van Mieghem T, Deprest J, Ghidini A, Chitty LS (2018) In case you missed it: the prenatal diagnosis editors bring you the most significant advances of 2017. Prenat Diagn 38(2):83-90. https://doi.org/10.1002/pd.5210

MacLeod R, Tibben A, Frontali M, Evers-Kiebooms G, Jones A, Martinez-Descales A, Roos RA, Editorial C, Working Group 'Genetic Testing Counselling' of the European Huntington Disease N (2013) Recommendations for the predictive genetic test in Huntington's disease. Clin Genet 83(3):221-231. https://doi.org/10.1111/j.1399-0004.2012.01900.x

Massaro G, Mattar CNZ, Wong AMS, Sirka E, Buckley SMK, Herbert BR, Karlsson S, Perocheau DP, Burke D, Heales S, Richard-Londt A, Brandner S, Huebecker M, Priestman DA, Platt FM, Mills K, Biswas A, Cooper JD, Chan JKY, Cheng SH, Waddington SN, Rahim AA (2018) Fetal gene therapy for neurodegenerative disease of infants. Nat Med 24(9):1317-1323. https://doi.org/10.1038/s41591018-0106-7

McMahan J (2013) Causing people to exist and saving people's lives. J Ethics 17(1-2):5-35

Mellis R, Chandler N, Chitty LS (2018) Next-generation sequencing and the impact on prenatal diagnosis. Expert Rev Mol Diagn 18(8):689-699. https://doi.org/10.1080/14737159.2018.1493924

Mills CA (2003) The child's right to an open future? J Soc Philos 34(4):499-599

Millum J (2014) The foundation of the child's right to an open future. J Soc Philos 45(4):522-538. https:// doi.org/10.1111/josp. 12076

Moldenhauer JS, Flake AW (2019) Open fetal surgery for neural tube defects. Best Pract Res Clin Obstet Gynaecol 58:121-132. https://doi.org/10.1016/j.bpobgyn.2019.03.004

Monaghan KG, Leach NT, Pekarek D, Prasad P, Rose NC, Practice AP, Guidelines C (2020) The use of fetal exome sequencing in prenatal diagnosis: a points to consider document of the American College of Medical Genetics and Genomics (ACMG). Genet Med. https://doi.org/10.1038/s41436-019-07317

Narayanan S, Blumberg B, Clayman ML, Pan V, Wicklund C (2018) Exploring the issues surrounding clinical exome sequencing in the prenatal setting. J Genet Counsel 27(5):1228-1237. https://doi.org/ 10.1007/s10897-018-0245-5

Parfit D (1984) Reasons and persons. Clarendon Press, Oxford

Richardson A, Ormond KE (2018) Ethical considerations in prenatal testing: genomic testing and medical uncertainty. Semin Fetal Neonatal Med 23(1):1-6. https://doi.org/10.1016/j.siny.2017.10.001

Roberts MA, Wasserman DT (Hrsg) (2009) Harming future persons. Ethics, genetics and the nonidentity problem. International library of ethics, law, and the new medicine, Bd. 35. Springer, Dordrecht

Rossidis AC, Stratigis JD, Chadwick AC, Hartman HA, Ahn NJ, Li H, Singh K, Coons BE, Li L, Lv W, Zoltick PW, Alapati D, Zacharias W, Jain R, Morrisey EE, Musunuru K, Peranteau WH (2018) In utero CRISPR-mediated therapeutic editing of metabolic genes. Nat Med 24(10):1513-1518. https:// doi.org/10.1038/s41591-018-0184-6

Schmitz D (2013) A new era in prenatal testing: are we prepared? Med Health Care Philos 16(3):357-364. https://doi.org/10.1007/s11019-012-9411-y

Schmitz D (2019) Why public funding for non-invasive prenatal testing (NIPT) might still be wrong: a response to Bunnik and colleagues. J Med Ethics. https://doi.org/10.1136/medethics-2019-105885

Schneider H, Faschingbauer F, Schuepbach-Mallepell S, Korber I, Wohlfart S, Dick A, Wahlbuhl M, Kowalczyk-Quintas C, Vigolo M, Kirby N, Tannert C, Rompel O, Rascher W, Beckmann MW, Schneider P (2018) Prenatal correction of x-linked hypohidrotic ectodermal dysplasia. N Engl J Med 378(17):1604-1610. https://doi.org/10.1056/NEJMoa1714322

Stapleton G, Dondorp W, Schroder-Back P, de Wert G (2019) Just choice: a Danielsian analysis of the aims and scope of prenatal screening for fetal abnormalities. Med Health Care Philos. https://doi.org/10. 1007/s11019-019-09888-5

Steigleder K (1998) Müssen wir, dürfen wir schwere (nicht-therapierbare) genetisch bedingte Krankheiten vermeiden? In: Düwell M, Mieth D (Hrsg) Ethik in der Humangenetik. Die neueren Entwicklungen der genetischen Frühdiagnostik aus ethischer Perspektive. Francke, Tübingen, S 91-119

Unruh C (2016) Present rights for future generations. Kriterion 30(3):77-92

Wadrup F, Holden S, MacLeod R, Miedzybrodzka Z, Nemeth AH, Owens S, Pasalodos S, Quarrell O, Clarke AJ, Consortium UKHs DP (2019) A case-note review of continued pregnancies found to be at a high risk of Huntington's disease: considerations for clinical practice. Eur J Hum Genet 27(8):1215-1224. https://doi.org/10.1038/s41431-019-0375-8

Wenstrom KD, Carr SR (2014) Fetal surgery: principles, indications, and evidence. Obstet Gynecol Clin North Am 124(4):817-835. https://doi.org/10.1097/AOG.0000000000000476 